\title{
Analysis signal transitions characteristics of BGA-via multi-chip module Baolin Zhou ${ }^{1, a}$, Dejian Zhou ${ }^{1, b}$ \\ ${ }^{1}$ Electromechanical Engineering College, Guilin University of Electronic Technology, Guilin 541004, China

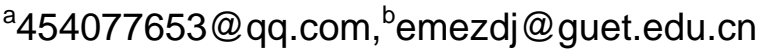

Keyword: structural parameters, BGA solder joint, vertical via, return loss

\begin{abstract}
BGA-via multi-chip module (MCM) was built by HFSS software, the return loss and insertion loss of MCM were obtained with different frequencies. The impacts of structure parameters included BGA radius, shield ring radius, the bonding pad radius and vertical hole apart from the shield hole distance on the return loss were studied. Studying results show that within a certain range, the return loss of BGA-via firstly increases then decreases as the increase of BGA radius, and the return loss decreases as the increase of shield ring radius, the anti-pad radius and vertical hole apart from the shield hole distance.
\end{abstract}

\section{Introduction}

As the rapid development of microwave technology, electronic equipment developed to the directions such as short, thin, high speed, high reliability and multi-function and low cost. Microwave multichip module(MCM) compared with traditional microwave devices, having small size, high integration, low power consumption, high stability and strong anti-interference advantages are widely used in wireless communication fields such as radar ,mobile phones[1]. But as the density of chip components and clock frequency of the signal increases, its interconnection structure caused by the parasitic effect and reflection, crosstalk, loss and other signal transmission problem are becoming more and more serious, has become the bottleneck of restricting components overall performance[2]. BGA-via connected as the main form of multiple chip component interconnection structure, is the most common form of transmission line connection between different layers[3], and has the advantages of high density of interconnection, high frequency features prominent, small package size, thermal expansion, high reliability and can realize wafer level packaging[4]. At low frequency, parasitic effects produced by hole is very small, basically didn't affect the transmission of signals. But with the increase of data rate and the signal to rise along the steepening, into the microwave band, the hole becomes a kind of "discrete structure", because the signal parasitic effects with hole, signal through a hole in the high frequency component part will produce attenuation and reflection, cause the distortion of signal, resulting in a series of signal integrity and electromagnetic interference problems. If we cann't guarantee the effective transmission of the signal under the condition of high speed and high frequency, will cause the loss of the whole system performance and even crash, influence on the reliability of the system. Therefore, established BGA-via 3D simulation model, and analyzed the main structural parameters affecting the signal transmission performance and the influence law is necessary.

\section{BGA - via 3D transition structure electromagnetic simulation model}

This article analyzed the BGA-via transition structure as shown in figure 1[5], A is BGA solder ball, $\mathrm{B}$ is the vertical hole. Front view and top view as shown in figure 2. BGA-via bottom material is the Ferro-A6 series of LTCC (low temperature co-firing ceramic), a relative permittivity value of 5.9, a loss tangent of 0.0027 , and each layer of tape thickness is $127 \mu \mathrm{m}$, sintering thickness of 0.6 $\mathrm{mm}$. Upper material uses R04350, a relative permittivity value of 3.58, the thickness is $1 \mathrm{~mm}$. Signal using coplanar waveguide and micro-strip lines by the vertical hole connection, takes the form of BGA welding between upper and lower two layers, a shielding hole around the signal transmission through hole was designed, not only used as a physical support, have the effect of 
shielding signal at the same time[6]. In order to ensure that a single coplanar waveguide mode spread along the line, two rows of shielding hole was set up between the land of coplanar waveguide[7-8]. For the detailed dimension parameters in the model: solder ball diameter of 0.3 $\mathrm{mm}$, solder ball height of $0.24 \mathrm{~mm}$, welding plate diameter of $0.18 \mathrm{~mm}$, anti-pad diameter of 0.65 $\mathrm{mm}$, shielding ring diameter of $1.4 \mathrm{~mm}$. All the vias were punched with a diameter of $0.15 \mathrm{~mm}$. The signal line and the signal-to-ground gap widths are $0.25 \mathrm{~mm}$ and $0.15 \mathrm{~mm}$ respectively.

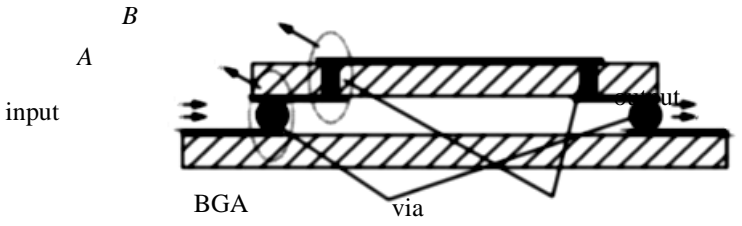

Fig.1 BGA RF transmission interconnection structure diagram

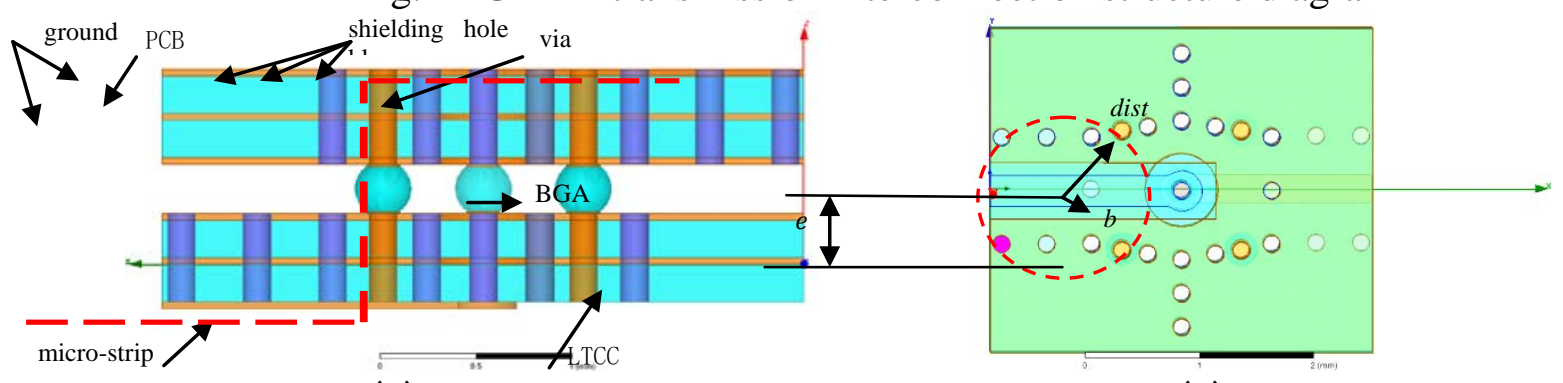

(a) front view

(b) top view

Fig.2 BGA-via interconnection model

BGA -via interconnection model was built by 3D electromagnetic field simulation software HFSS, and the model is regarded as two port network, set up the conditions of boundary and convergence and port was added on both ends of the coplanar waveguide and micro-strip line. The grid software adaptive subdivision was adapted, and $0 \sim 40$ GHZ frequency characteristic are analyzed.

\section{The BGA-via transmission characteristics analysis of the simulation model}

Analyzing return loss of simulation model with different BGA radius. When the transmission characteristics of BGA-via interconnection model was analyzed, fixed the anti-pad radius $b$ of 0.325 $\mathrm{mm}$, shielding ring radius dist of $0.7 \mathrm{~mm}$, the shield hole to via distance $e$ of $0.615 \mathrm{~mm}$, selection of BGA radius $r 0.15,0.225,0.3$ and $0.375 \mathrm{~mm}$. The model of different radius of BGA solder ball were built through HFSS simulation software, and the return loss of the different radius of BGA solder ball were gained.

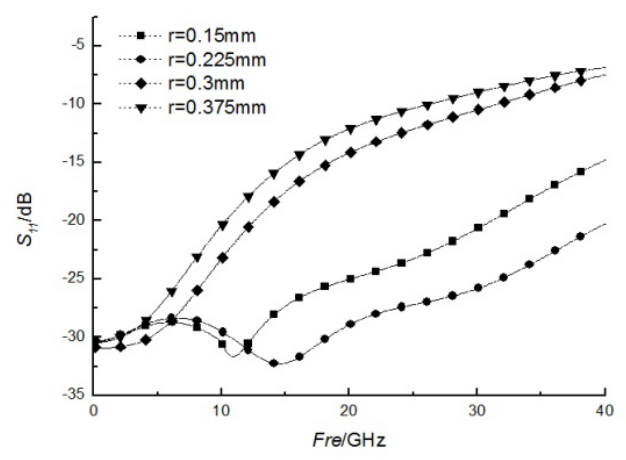

Fig.3 $S_{11}$ curves of different BGA solder ball diameters

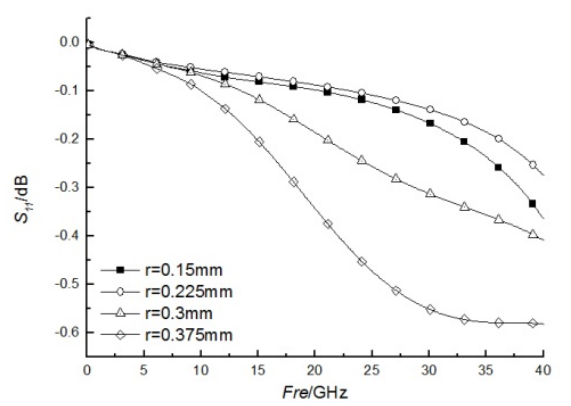

Fig.4 $S_{21}$ curves of different BGA solder ball diameters

Figures 3 and 4 shows different radius of BGA solder ball S11 and S21 graph. In figure 3, from top to bottom S11 curve corresponding to the BGA radius $r$ is $0.375,0.3,0.15,0.225 \mathrm{~mm}$, and at 40 $\mathrm{GHZ}$, order of them the return loss is $7.4,7.48,14.8,20.27 \mathrm{~dB}$. In figure $4 \mathrm{~S} 21$ curve from top to bottom corresponds to the BGA radius $r$ is $0.225,0.15,0.3,0.375 \mathrm{~mm}$, and at $40 \mathrm{GHZ}$ their insertion loss of $0.28,0.36,0.41,0.58 \mathrm{~dB}$. When BGA radius $r$ is $0.225 \mathrm{~mm}$, transmission 
coefficient reduced $0.28 \mathrm{~dB}$ within $0 \sim 40 \mathrm{GHZ}$, and when $r$ is $0.375 \mathrm{~mm}$ transmission coefficient reduced $0.58 \mathrm{~dB}$ within $0 \sim 40 \mathrm{GHZ}$, relative decline is $52 \%$, it can be seen that BGA radius has much effect on the insertion loss. As can be seen from the figure 3 and figure 4, within the $5 \mathrm{GHZ}$, different radius of BGA solder ball have less effect on the return loss. With the increase of frequency, BGA radius of connected components of transmission performance influence is more and more obvious. Within a certain range, with the increase of BGA radius growing insertion loss and return loss is more and more small, that is, signal transmission energy to the receiver is more and more less, signal transmission performance is becoming more and more poor.

Analyzing return loss of simulation model with different anti-pad radius. Selected anti-pad radius of $0.3,0.325,0.35$ and $0.375 \mathrm{~mm}$. The above four different radius of anti-pad after modeling and simulation results are shown in figure 5.

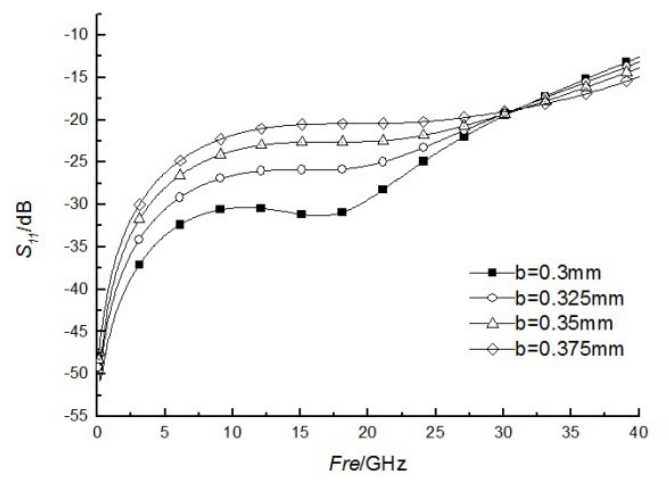

Fig.5 $S_{11}$ curves of different anti-pad radii

Figure 5 is the return loss curve of different anti-pad radius. In figure 5, from top to bottom S11 curve corresponding to the anti-pad is $0.375,0.35,0.325,0.3 \mathrm{~mm}$. The figure shows that S11 curve in $30 \mathrm{GHZ}$ have a meet. Vertical hole itself exists parasitic capacitance and inductance of the ground or power in the high-speed circuit, according to the literature [9] formula, with the increase of the radius of anti-pad, the hole of parasitic capacitance decreases, the impedance of the circuit $\mathrm{R}$ $=\mathrm{L} / \mathrm{C}$ is gradually increasing, so the return loss will gradually increase and transmission performance poor. As can be seen from the figure 5, within $0 \sim 30 \mathrm{GHZ}$, when the anti-pad radius $b$ of $0.3 \mathrm{~mm}$ the return loss is minimum, transmission performance is the best. When b of $0.375 \mathrm{~mm}$ return loss is the largest, the transmission characteristics is the worst. Within 30 to $40 \mathrm{GHZ}$, return loss and before the opposite trend. Because when the signal frequency increases, the hole will lower characteristic impedance of transmission line, lead to the impedance mismatch, causing the phenomenon such as reflection, crosstalk noise. As can be seen from S parametric curves, when the other parameters as constant value within a certain range S11 parameter increases with the increase of the radius of anti-pad $b$, S11 reflected the reflection loss, namely, $b$ is larger, the reflection loss greater.

Analyzing return loss of simulation model with different shielding ring radius. Selected shielding ring radius of $0.5,0.7,0.9$ and $1.1 \mathrm{~mm}$. The above four different radius of shielding ring after modeling and simulation results are shown in figure 6.

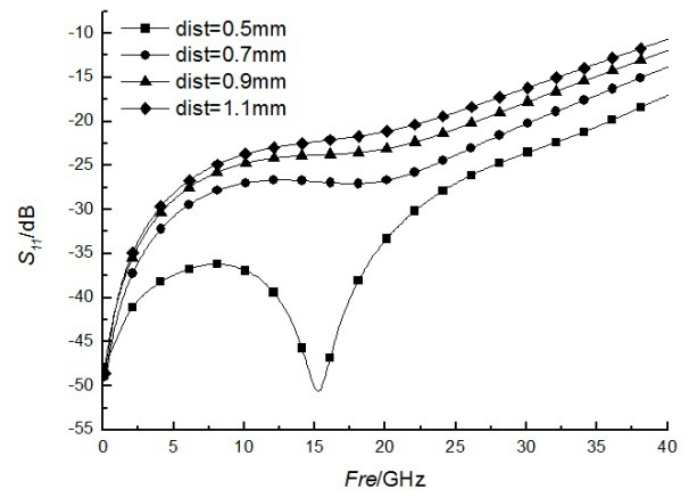

Fig.6 $S_{11}$ curves of different shield ring radii 
Figure 6 is the return loss curve of different shielding ring radius. In figure 6, from top to bottom S11 curve corresponding to the shielding ring radius is 1.1, 0.9, 0.7, $0.5 \mathrm{~mm}$. As can be seen from the figure of return loss increases with the increase of shield ring radius in a certain range, return loss minimum when $d$ is $0.5 \mathrm{~mm}$, and the resonance phenomenon around $15 \mathrm{GHZ}$, reflection is the most serious when $d$ is $1.1 \mathrm{~mm}$, the return loss is the largest. So the radius of the shield ring of 0.5 mm impedance continuity is better, the transmission coefficient is larger.

Analyzing return loss of simulation model with different vertical hole to hole distance shield. Selected vertical hole to hole distance shield of $0.365,0.49,0.615$ and $0.74 \mathrm{~mm}$. The above four different vertical hole to hole distances shield after modeling and simulation results are shown in figure 7.

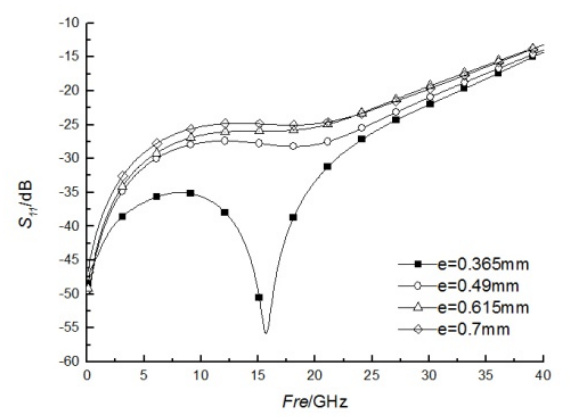

Fig7 $S_{11}$ curve of different vertical hole to hole distance shield

Figure7 is the return loss curve of different vertical hole to hole distances shield. In figure 7, from top to bottom S11 curve corresponding to the vertical hole to hole distance shield $e$ is 0.365 , $0.49,0.615,0.74 \mathrm{~mm}$. As can be seen from the figure, within $0 \sim 20 \mathrm{GHZ}$ return loss increase gradually with the increase of distance $e$, Transmission performance weakened, resonance point was appeared when the distance $e$ of $0.365 \mathrm{~mm}$ around $15 \mathrm{GHZ}$ frequency, transmission characteristics is the best. Extended $20 \mathrm{GHZ}$, spacing of $0.615 \mathrm{~mm}$ and $0.74 \mathrm{~mm}$ of return loss values were similar, because with the increase of distance $e$, the effect of shielding hole gradually diminish.

\section{Conclusion}

Through the above analysis, we can get the following basic conclusions:

In the other conditions unchanged, only change under the condition of a certain factor, the return loss of BGA-via firstly increases then decreases as the increase of BGA radius, and the return loss decreases as the increase of shield ring radius, the anti-pad radius and vertical hole apart from the shield hole distance.

Under the condition of the process allows to use a smaller radius of solder ball, and makes the shielding ring and block shielding hole near the transmission signal, so that not only can obtain better signal transmission performance, and for such high density microwave multichip module, small solder ball also leave more space layout for circuit design.

\section{Acknowledgements}

This work was supported by graduate student innovation project“Based on TMS320LF240x photoelectric device for device design”.

\section{Reference}

[1]Shuai Yao. Investigation on Microwave Characteristics of gold wire bonding and Via Interconnects Based on LTCC Technology(Master.D, Xian university of electronic science and technology,2012). [2]IAN R, STEPAN L. Rf microwave monolithic integrated circuit technology and design [M].G.J.Wen. Beijing: Electronic industry press,2007:35-69.

[3]Xin Xu. Transition of LTCC Millimeter Wave Integrated Transmission Line and Parasitic Mode 
Analysis and Modeling(Master.D, University of electronic science and technology,2009).

[4]Yin Liang,Xunchao Lin. Signal integrity analysis of TSV based on orthogonal design[J].Electronic components and materials,2015,34(5):66-70.

[5]Jun Zhou,Wenbin Dou. Broadband Vertical Transition between Substrates in Application of SIP Technology[J].2012,32(1):36-39.

[6]Qixia Zhang. Microwave LTCC Vertical Hole Interconnect Modeling Research (Master.D, University of electronic science and technology,2012).

[7] KANGASVIERI T, HALME J, LAHTI M. Ultra-wideband shield vertical via transitions from DC up to the V-band[C]//Proceedings of the European Microwave Integrated Circuits Conference.Manchester,UK:The Conference Organizer, 2006.

[8] CHEN H F,LI Q,TSANG L,et al.Coupling of large number of vias in electronic packaging structures and differential signaling [C]//IEEE MTT-S Digest. Seattle, WA, USA:IEEE,2002.

[9]Lili Wei,Hao Liu. Research of Via in High speed PCB Design[J].Printed Circuit Information,2007(9):31-33. 